\title{
Symptom burdens related to chemotherapy-induced anemia in stage IV cancer
}

\author{
Leila Family, PhD, ${ }^{a}$ Hairong Xu, PhD, ${ }^{\mathrm{b}}$ Kim Cannavale, MPH, ${ }^{\mathrm{c}}$ Bhakti Mehta, MD, ${ }^{\mathrm{b}}$ \\ Andrew Pourmoussa, MD, ${ }^{d}$ Lanfang $\mathrm{Xu}, \mathrm{MS},{ }^{\mathrm{e}}$ and Chun Chao, $\mathrm{PhD}^{\mathrm{c}}$
}

${ }^{a}$ Office of Health Assessment and Epidemiology, Los Angeles County Department of Public Health, Los Angeles, California, ${ }^{\mathrm{b} A m g e n ~ I n c, ~ T h o u s a n d ~ O a k s, ~ C a l i f o r n i a, ~ ' D e p a r t m e n t ~ o f ~ R e s e a r c h ~ a n d ~ E v a l u a t i o n, ~ K a i s e r ~ P e r m a n e n t e ~ S o u t h e r n ~ C a l i f o r n i a, ~}$ Pasadena, California, dDepartment of Medicine, Kaiser Permanente Los Angeles Medical Center, Los Angeles, California, ${ }^{\mathrm{e}}$ MedHealth Statistical Consulting Inc, Solon, Ohio

Background Chemotherapy-induced anemia $(\mathrm{CIA})$ is associated with many symptoms that negatively impact quality of life. However, a systematic examination of symptoms in patients with CIA is lacking.

Objective To describe the occurrence of a comprehensive list of symptoms in patients with stage IV malignancies by CIA status. Methods Patients diagnosed with stage IV non-Hodgkin lymphoma, breast, and lung cancer at Kaiser Permanente Southern California (2010-2012) were eligible. CIA was defined as hemoglobin $<10 \mathrm{~g} / \mathrm{dL}$ after the initiation of chemotherapy.

Standardized record review evaluated the occurrence of symptoms for all patients who developed CIA $(n=402)$, and a random sample of patients who did not develop CIA $(n=98)$. The prevalence of each symptom and the distribution of number of symptoms per patient were described overall and by anemia grade.

Results Mean number of symptoms during chemotherapy for patients who did and did not develop CIA was 6.8 and 4.1 , respectively $(P<.01)$. Fatigue $(90 \%)$, dyspnea or shortness of breath $(58 \%)$, nausea or vomiting $(56 \%)$, and loss of appetite $(56 \%)$ were documented in $>50 \%$ of patients who developed $\mathrm{CIA}$, whereas only fatigue $(77 \%)$ was noted in $>50 \%$ patients without $\mathrm{CIA}$. Several symptoms, including depression, diarrhea, dizziness or lightheadedness, and dyspnea, particularly demonstrated a clearly increasing prevalence with declining hemoglobin level. The mean number of symptoms per patient increased as $\mathrm{CIA}$ grade increased (3.6 symptoms for grade 2, and 5.4 symptoms for grades 3 and 4, respectively).

Limitations No causal relationship was examined due to descriptive design.

Conclusions High-grade CIA correlates with an increased symptom burden in patients with stage IV malignancies.

Funding Amgen Inc, maker of ESA used in the treatment of anemia

A nemia is a common complication of cancer treatment as well as of cancer itself. Most cancer patients undergoing chemotherapy experience anemia sometime during their treatment course. ${ }^{1,2}$ Moderate to severe anemia is associated with an array of symptoms that are known to compromise the physical functioning and quality of life of cancer patients. Common anemia-related symptoms include fatigue, drowsiness, depression, dyspnea, tachycardia, and dizziness. ${ }^{1,3-7}$

Symptoms produced by cancer itself or the disease treatment (ie, side effects such as anemia) collectively compose a patient's symptom burden. ${ }^{8}$ Although the occurrence of anemia-related fatigue has been described more systematically, other clini- cal presentations of chemotherapy-induced anemia (CIA) are not well characterized. Furthermore, the overall symptom burdens associated with different ranges of hemoglobin $(\mathrm{Hb})$ concentrations have also not been well reported. Although various tools have been developed to facilitate the reporting of fatigue and other symptoms experienced by patients with CIA, such as the Functional Assessment of Cancer Therapy-Anemia (FACT-An) questionnaire and the MD Anderson Symptom Inventory (MDASI), ${ }^{9-11}$ these questionnaires have not been extensively used outside of the research context. As such, knowledge on symptom burdens associated with CIA in realworld patient populations remains lacking.

Given the common occurrence of CIA, manage-

Accepted for publication October 26, 2018. Correspondence: Chun Chao, PhD; Chun.R.Chao@kp.org. Disclosures: Hairong Xu and Bhakti Mehta were employees of Amgen during study execution and received salary and stock as part of employment. Amgen provided research support to Kaiser Permanente to perform this study who, in turn, employed or contracted with the non-Amgen authors. Authors did not report any other potential conflicts of interest. JCSO 2018; 16(4):e260-e271. (C2018 Frontline Medical Communications. doi: https://doi.org/10.12788/jcso.0432 
ment of CIA and associated symptoms plays an important role to patients' quality of life during cancer treatment. Symptom control is often the main goal for patients with stage IV cancers, as treatment for disease is most likely palliative or noncurative. To facilitate supportive care planning, it is important to understand patient symptom burdens as chemotherapy progresses over cycles and $\mathrm{Hb}$ levels decline. We conducted a comprehensive medical record review study in patients diagnosed with stage IV nonHodgkin lymphoma (NHL), breast cancer, and lung cancers at Kaiser Permanente Southern California (KPSC), a large community-based health care delivery system. The objective of this study was to report the occurrence of CIArelated symptoms throughout the course of chemotherapy and by $\mathrm{Hb}$ levels.

\section{Methods \\ Study setting and population}

KPSC is an integrated managed-care organization that provides comprehensive health services for 4 million racially, ethnically, and socioeconomically diverse members who broadly represent the population in Southern California. ${ }^{12}$ The organization maintains electronic records of health care received by its members, including physician record notes and clinical databases such as laboratory test results, diagnosis codes, medical procedures, medication dispenses, and disease registries. KPSC's cancer registry is Surveillance, Epidemiology, and End Results, which is affiliated and routinely collects information on age, sex, race and/or ethnicity, cancer type, histology, and stage at diagnosis.

Patients who met the following inclusion criteria were included in this study: diagnosed with stage IV NHL, breast cancer, or lung cancer at age 18 years or older at KPSC between March 25, 2010 and December 31,2012; initiated myelosuppressive chemotherapy at KPSC before June 30, 2013 (only the first chemotherapy course was included in this evaluation); and had at least $1 \mathrm{Hb}$ measurement during the course of chemotherapy. Of those who met the inclusion criteria, patients who met the following criteria were excluded if they had less than 12 months KPSC membership before start of chemotherapy, missing information on cancer stage or chemotherapy regimen/agents, a diagnosis of myelodysplastic syndrome before chemotherapy initiation, a diagnosis of inherited anemia, an $\mathrm{Hb}$ concentration $<10 \mathrm{~g} / \mathrm{L}$ within 3 months before chemotherapy initiation, a transfusion within 2 weeks before chemotherapy initiation, radiation within 4 months before chemotherapy initiation, or bone marrow transplantation within 12 months before chemotherapy initiation or during the chemotherapy course. These exclusion criteria were applied to evaluate symptom burdens most likely related to CIA as opposed to other cancer treatment or pre-existing anemia.

CIA in this study was defined as moderate to severe anemia with $\mathrm{Hb}<10 \mathrm{~g} / \mathrm{dL}$ after chemotherapy initiation. Based on this definition for CIA, all patients who developed CIA between the first chemotherapy administration to 60 days after the last dose of chemotherapy were included for the record review. In addition, a random sample of 100 patients who did not develop CIA (ie, did not reach an $\mathrm{Hb}<10 \mathrm{~g} / \mathrm{dL}$ during chemotherapy) but otherwise met study eligibility criteria was also reviewed to serve as a comparison group. Of those, 2 patients were subsequently excluded after record review because of findings of ineligibility, so only 98 patients were presented. The large number of patients (ie, >4,000) who did not develop CIA made record review of all patients infeasible.

\section{Data collection}

Data on anemia-related symptoms or signs and anemiarelated comorbidities (Table 1 ) were collected by standardized review of physician record notes in the electronic medical records. A set of 24 anemia-related symptoms were identified based on the literature and clinical expertise and included abdominal pain, blurred vision/double vision/loss of vision, cold intolerance/coldness in hands or feet, depression/anxiety, diarrhea, dizziness/lightheadedness, dyspnea/ shortness of breath/tachypnea, edema, fatigue, headache, heart failure, heat intolerance, hypotension, insomnia, leg pain, loss of appetite, nausea/vomiting, pale skin, palpitations/tachycardia, paralysis/ataxia/numbness or tingling in extremities, pectoral angina/chest pain, sweating/diaphoresis, syncope, and vertigo. Record review period was defined as 1 month before chemotherapy to 60 days after the last dose of chemotherapy in the first course. To understand the development of new symptoms during chemotherapy treatment, pre-existing symptoms documented within 1 month before chemotherapy initiation were recorded. The entire record review process was standardized between 2 trained abstractors, including the training, instruction manual, ongoing feedback, abstraction form/database, and coding.

The data elements extracted included the date the symptom was documented, date the symptom started, symptom duration (when available), and any relevant comments regarding the symptom (ie, if dyspnea was at rest or on exertion, whether the symptom was a side effect caused by chemotherapy, or change in symptom severity). Ten percent of the records were reviewed independently by 2 abstractors to ensure quality control. Additional quality control measures included SAS algorithms (SAS Institute, Inc., Cary, North Carolina) to check reasonability and logical consistency in the abstracted data.

Patient demographic characteristics, cancer stage, additional selected comorbidities (Table 1), chemotherapy information, $\mathrm{Hb}$ test results, and anemia treatment, including erythrocyte stimulating agent (ESA) use and red blood cell transfusion, were collected using KPSC's cancer regis- 
TABLE 1 Distribution of patient demographic and clinical characteristics

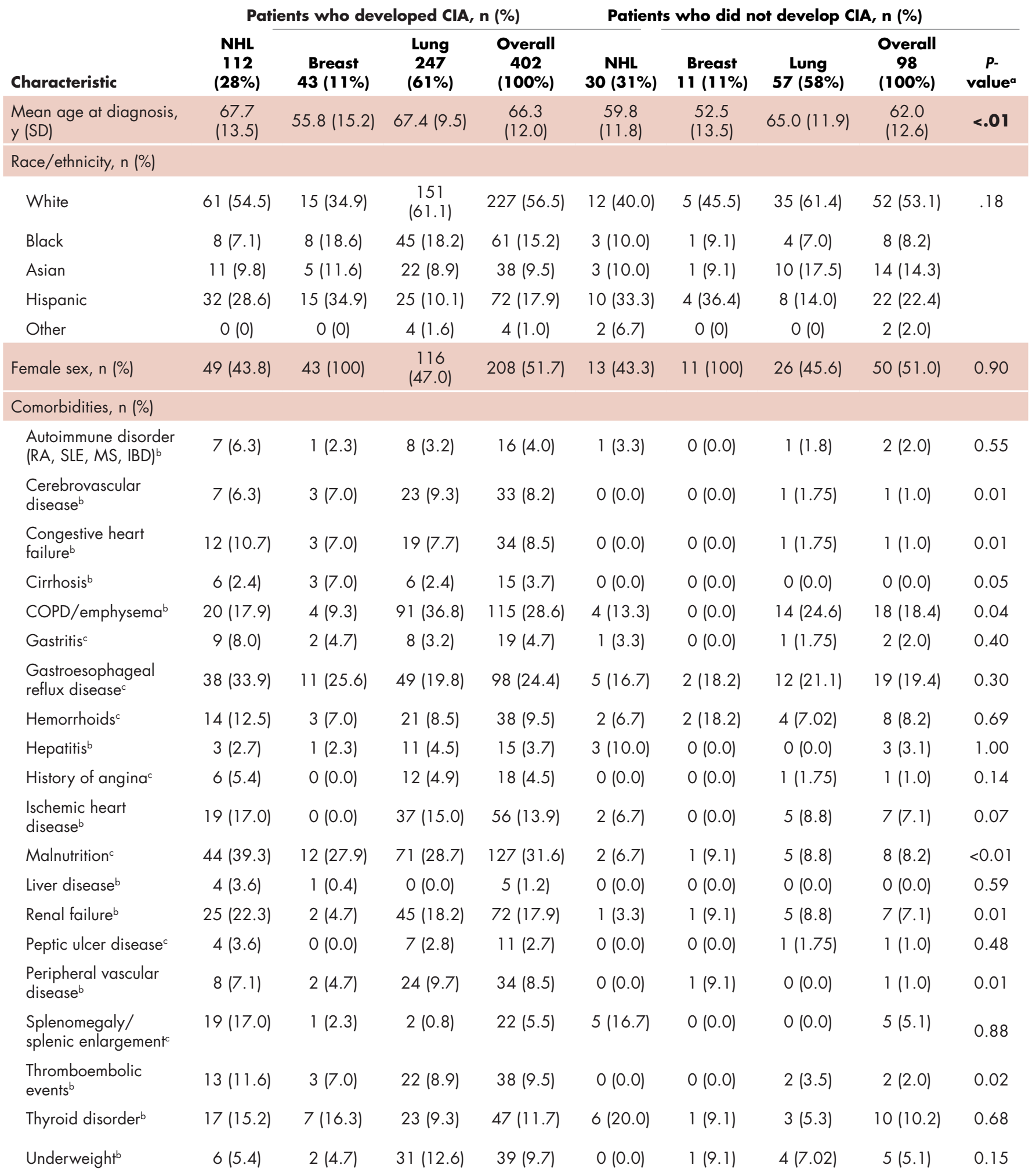




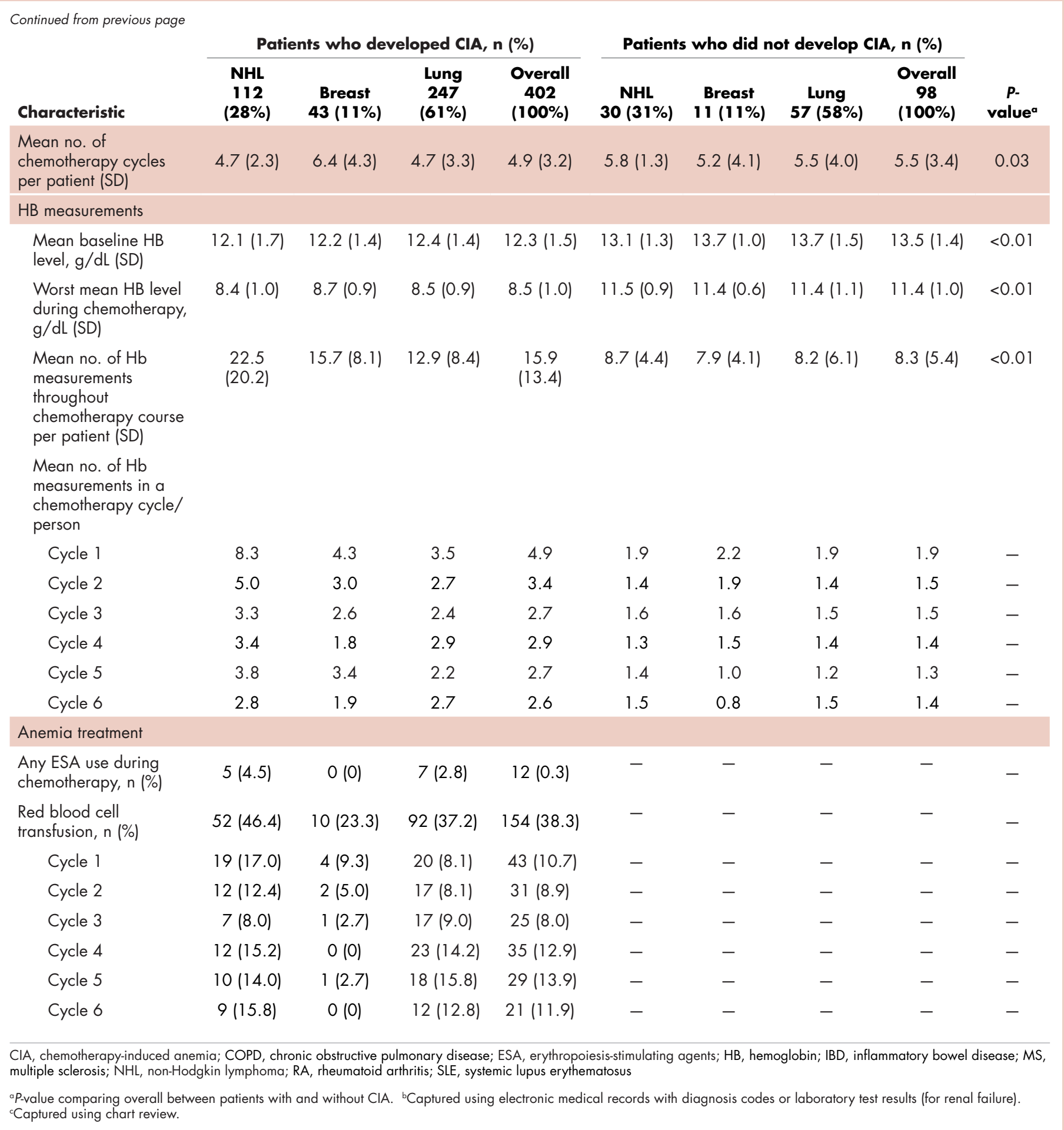

try and clinical databases. Anemia was defined by severity as grade 1 (10 g/dL to lower limit of normal, ie, $14 \mathrm{~g} / \mathrm{dL}$ for men and $12 \mathrm{~g} / \mathrm{dL}$ for women), grade $2(8.0-9.9 \mathrm{~g} / \mathrm{dL})$, grade $3(6.5-7.9 \mathrm{~g} / \mathrm{dL})$, and grade $4(<6.5 \mathrm{~g} / \mathrm{dL})$ following the National Cancer Institute's Common Terminology Criteria for Adverse Events. ${ }^{13}$

\section{Statistical analysis}

Distributions of demographic, cancer, and treatment characteristics were calculated by CIA status, overall and by cancer type. Differences between patients who did and did not develop CIA were assessed using chi-square test and Kruskal-Wallis test. For those who developed CIA, the dis- 
TABLE 2 Number of anemia episodes by anemia grade in each chemotherapy cycle for patients who developed chemotherapy-induced anemia

Patients with anemia episode, $\mathbf{n}(\%)^{a}$

\begin{tabular}{ccccccc} 
Grade & $\begin{array}{c}\text { Cycle 1 } \\
(\mathbf{n = 4 0 2 )}\end{array}$ & $\begin{array}{c}\text { Cycle 2 } \\
(\mathbf{n}=\mathbf{3 4 7})\end{array}$ & $\begin{array}{c}\text { Cycle 3 } \\
(\mathbf{n = 3 1 4 )}\end{array}$ & $\begin{array}{c}\text { Cycle 4 } \\
(\mathbf{n = 2 7 1 )}\end{array}$ & $\begin{array}{c}\text { Cycle 5 } \\
(\mathbf{n}=\mathbf{2 0 9})\end{array}$ & $\begin{array}{c}\text { Cycle 6 } \\
(\mathbf{n}=\mathbf{1 7 6})\end{array}$ \\
\hline $1^{\mathrm{b}}$ & $197(56.6)$ & $172(54.8)$ & $142(49.3)$ & $96(38.4)$ & $81(40.9)$ & $70(48.6)$ \\
\hline $2^{\mathrm{b}}$ & $119(34.2)$ & $121(38.5)$ & $124(43.1)$ & $129(51.6)$ & $94(47.5)$ & $60(41.7)$ \\
\hline $3^{\mathrm{b}}$ & $29(8.3)$ & $19(6.1)$ & $22(7.6)$ & $22(8.8)$ & $22(11.1)$ & $12(8.3)$ \\
\hline $4^{\mathrm{b}}$ & $3(0.9)$ & $2(0.6)$ & $0(0)$ & $3(1.2)$ & $1(0.5)$ & $2(1.4)$
\end{tabular}

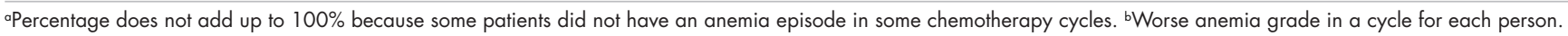

tribution of the worst anemia grade was also calculated for each cycle of chemotherapy.

Next, the distributions for the following symptom categories were calculated in the 2 study samples defined by CIA status: pre-existing symptoms that occurred before chemotherapy, any symptoms during chemotherapy (ie, whether they started before chemotherapy), and incident symptoms during chemotherapy (ie, new symptoms that only started after chemotherapy). Specifically, the proportion of patients with each individual symptom and the distribution of the number of symptoms per patient were calculated. Differences in symptom distribution by CIA status were assessed using chi-square test.

The distribution of symptoms in each chemotherapy cycle was calculated up to 6 chemotherapy cycles (as $>80 \%$ of the patients only had treatment up to 6 cycles) in the 2 study samples defined by CIA status. For this analysis, a symptom was "mapped" to a cycle if the date (or date range) of the symptom fell within the date range of that chemotherapy cycle. In patients who developed CIA, the distribution of symptoms was also calculated by anemia grade. This was again done on the chemotherapy cycle level. For each chemotherapy cycle, an anemia grade was assigned (no anemia or anemia grade 1, 2, 3, and 4) using the lowest $\mathrm{Hb}$ measurement in that cycle. Symptoms that occurred in a chemotherapy cycle were then "mapped" to the anemia grade of that cycle. Some patients had more than 1 anemia event of the same grade (eg, if a patient's grade 2 anemia persist across cycles). For these patients, we randomly selected only 1 anemia event of the same grade from each patient to be included in this analysis. Patients could still contribute multiple events of different grades to this analysis. We calculated the mean number of symptoms per patient for each anemia grade (ie, 1-4) separately. Because of the small number of patients who developed grade 4 anemia $(n=11)$, they were combined with the grade 3 patients when the distributions of individual symptoms were evaluated.

All analyses were repeated stratified by gender. $P$ values for differences between men and women were calculated using chi-square test or $t$ test. All analyses were conducted using SAS version 9.3.

\section{Results}

A total of 402 stage IV NHL, breast, and lung cancer patients who developed CIA and 98 patients who did not develop CIA during the first course of chemotherapy were included (Figure 1). The distribution of cancer types in the study sample were similar across CIA status (Table 1). The mean age at diagnosis was 66 years in patients who developed CIA and 62 years in patients who did not develop CIA. Women accounted for half of the patients in both study samples (52\% and 51\%, respectively). Most of the study patients were of non-Hispanic white race/ethnicity. Chronic obstructive pulmonary disease/emphysema and gastroesophageal reflux disease were among the most common comorbidities examined in both study samples, while malnutrition and moderate to severe renal disease were also common in patients who developed CIA (Table 1).

The mean $\mathrm{Hb}$ level before chemotherapy was lower for patients who developed CIA compared with patients who did not develop CIA $(12.3 \mathrm{~g} / \mathrm{dL}$ and $13.5 \mathrm{~g} / \mathrm{dL}$, respectively; Table 1). The mean lowest $\mathrm{Hb}$ level during chemotherapy was $8.5 \mathrm{~g} / \mathrm{dL}$ for patients who developed CIA and $11.4 \mathrm{~g} / \mathrm{dL}$ for patients without CIA (Table 1). The number of anemia events by grade in each chemotherapy cycle in patients who developed CIA is shown in Table 2. Use of ESA was extremely rare in the study population. About $23 \%$ to $46 \%$ of patients who developed anemia received red blood cell transfusion throughout the chemotherapy cycles. There was no clear trend of use of red blood transfusion over cycles (Table 1).

Table 3 shows the number and proportion of study patients with each of the symptoms documented before and after chemotherapy initiation for the 2 study samples. Patients who developed CIA had statistically significantly more pre-existing symptoms, incident symptoms, or any symptoms that occurred during chemotherapy compared with patients who did not develop CIA. The mean number of pre-existing symptoms was 1.7 (standard deviation 


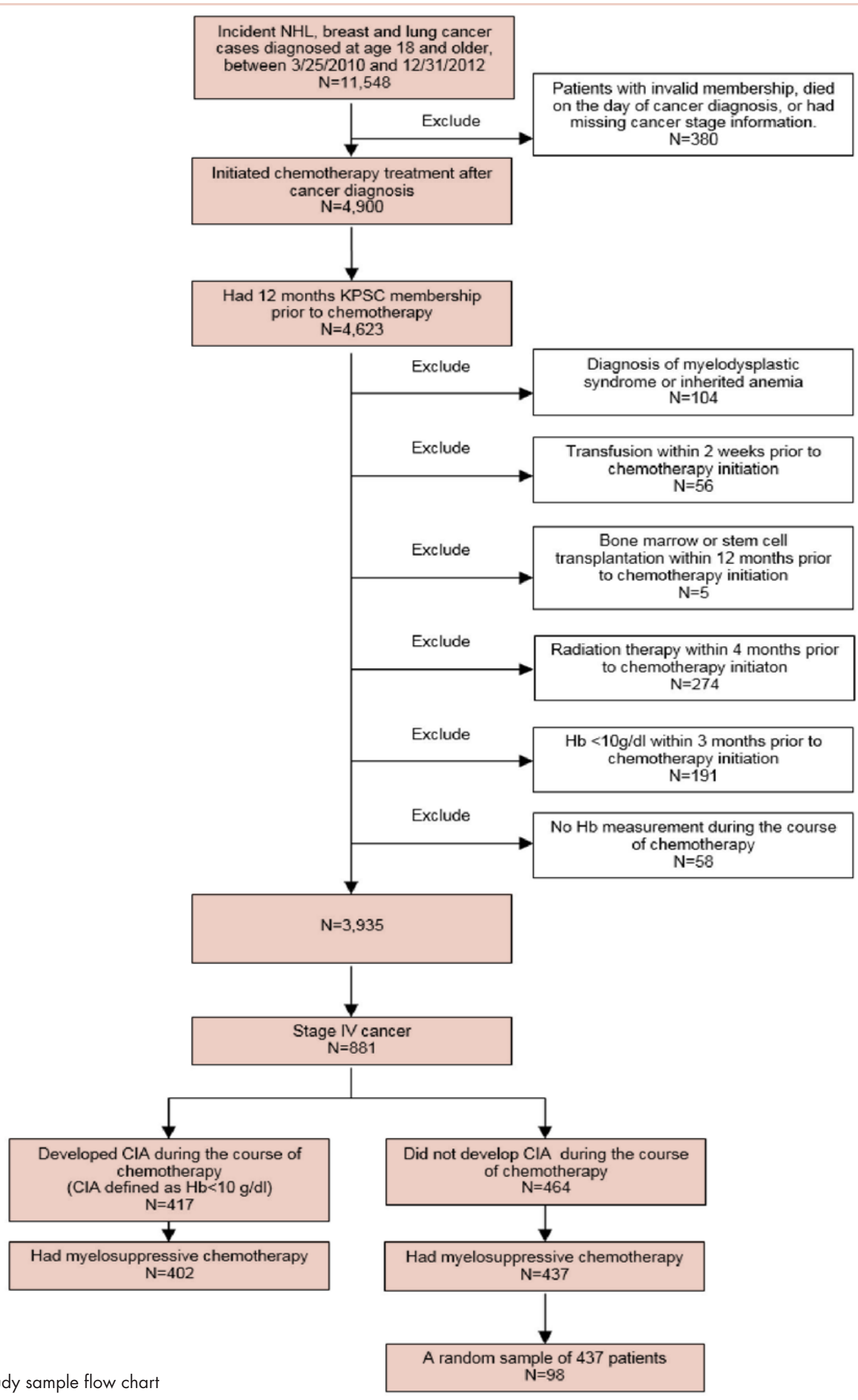


TABLE 3 Distribution of symptoms in patients who developed $\mathrm{ClA}$ and those who did not $(\mathrm{n}=98)$

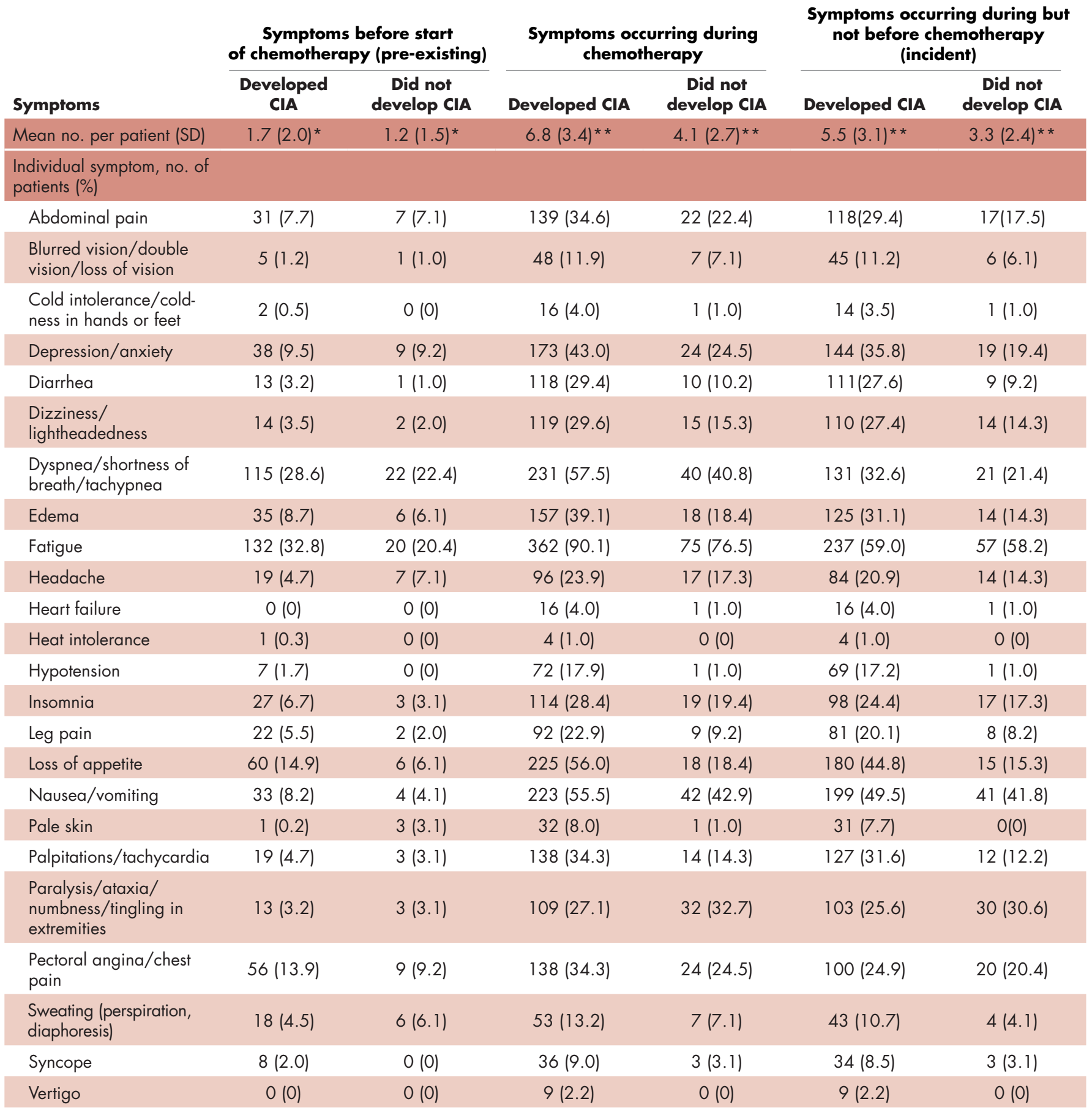

$\mathrm{CIA}$, chemotherapy-induced anemia

${ }^{*} P=.04 .{ }^{*} P<.01$.

[SD], 2.0) for those with CIA and $1.2(\mathrm{SD}, 1.5)$ for those without CIA $(P=.04)$. The mean number of symptoms that occurred during chemotherapy was $6.8(\mathrm{SD}, 3.4)$ and 4.1 (SD, 2.7), respectively $(P<.01)$. Of individual symp- toms, fatigue was the most commonly documented symptom during chemotherapy in patients who developed CIA, noted in $90 \%$ of the study sample (Table 3). Dyspnea/ shortness of breath (58\%), nausea/vomiting (56\%), and 


\begin{tabular}{|c|c|c|c|c|c|c|}
\hline \multicolumn{7}{|c|}{ Patients who developed CIA } \\
\hline Symptoms & $\begin{array}{l}\text { Cycle } 1 \\
(n=402)\end{array}$ & $\begin{array}{c}\text { Cycle } 2 \\
(n=347)\end{array}$ & $\begin{array}{l}\text { Cycle } 3 \\
(n=314)\end{array}$ & $\begin{array}{l}\text { Cycle } 4 \\
(n=271)\end{array}$ & $\begin{array}{l}\text { Cycle } 5 \\
(n=209)\end{array}$ & $\begin{array}{r}\text { Cycle } 6 \\
(n=176)\end{array}$ \\
\hline Mean no. per patient (SD) & $2.6(2.6)$ & $1.9(2.1)$ & $1.8(1.9)$ & $1.7(2)$ & $1.6(2)$ & $2.2(2)$ \\
\hline \multicolumn{7}{|l|}{$\begin{array}{l}\text { Individual symptom, no. of patients } \\
(\%)\end{array}$} \\
\hline Abdominal pain & 71 (17.7) & $35(10.1)$ & $26(8.3)$ & $25(9.2)$ & $20(9.6)$ & $27(15.3)$ \\
\hline $\begin{array}{l}\text { Blurred vision/double vision/ } \\
\text { loss of vision }\end{array}$ & $17(4.2)$ & $9(2.6)$ & $12(3.8)$ & $8(3.0)$ & $2(1.0)$ & $9(5.1)$ \\
\hline $\begin{array}{l}\text { Cold intolerance/coldness in hands } \\
\text { or feet }\end{array}$ & $7(1.7)$ & $5(1.4)$ & $4(1.3)$ & $2(0.7)$ & $0(0)$ & $1(0.6)$ \\
\hline Depression/anxiety & 79 (19.7) & $43(12.4)$ & $42(13.4)$ & $30(11.1)$ & 25 (12.0) & $38(21.6)$ \\
\hline Diarrhea & 51 (12.7) & $26(7.5)$ & $25(8.0)$ & $21(7.7)$ & $14(6.7)$ & $17(9.7)$ \\
\hline Dizziness/lightheadedness & $41(10.2)$ & $35(10.1)$ & $22(7.0)$ & $26(9.6)$ & $21(10.1)$ & $16(9.1)$ \\
\hline $\begin{array}{l}\text { Dyspnea/shortness of breath/ } \\
\text { tachypnea }\end{array}$ & $120(29.9)$ & $84(24.2)$ & $82(26.1)$ & $68(25.1)$ & $43(20.6)$ & $43(24.4)$ \\
\hline Edema & $76(18.9)$ & $46(13.3)$ & $46(14.6)$ & $30(11.1)$ & $22(10.5)$ & $29(16.5)$ \\
\hline Fatigue & $205(51.0)$ & $159(45.8)$ & $149(47.5)$ & $128(47.2)$ & $88(42.1)$ & $97(55.1)$ \\
\hline Headache & $54(13.4)$ & $27(7.8)$ & $16(5.1)$ & $16(5.9)$ & $12(5.7)$ & $15(8.5)$ \\
\hline Heart failure & $13(3.2)$ & $1(0.3)$ & $0(0)$ & $1(0.4)$ & $1(0.5)$ & $0(0)$ \\
\hline Heat intolerance & $3(0.7)$ & $0(0)$ & $1(0.3)$ & $0(0)$ & $0(0)$ & $0(0)$ \\
\hline Hypotension & $36(9.0)$ & $11(3.2)$ & $10(3.2)$ & $8(3.0)$ & 7 (3.3) & $6(3.4)$ \\
\hline Insomnia & $50(12.4)$ & $37(10.7)$ & 31 (9.9) & $15(5.5)$ & $12(5.7)$ & $17(9.7)$ \\
\hline Leg pain & $30(7.5)$ & $30(8.6)$ & $18(5.7)$ & $17(6.3)$ & $16(7.7)$ & $13(7.4)$ \\
\hline Loss of appetite & $124(30.8)$ & $62(17.9)$ & $51(16.2)$ & $44(16.2)$ & 27 (12.9) & 38 (21.6) \\
\hline Nausea/vomiting & $123(30.6)$ & $74(21.3)$ & $59(18.8)$ & $41(15.1)$ & $33(15.8)$ & $41(23.3)$ \\
\hline Pale skin & $15(3.7)$ & $6(1.7)$ & $3(1.0)$ & $5(1.9)$ & $4(1.9)$ & $2(1.1)$ \\
\hline Palpitations/tachycardia & $58(14.4)$ & $37(10.7)$ & $20(6.4)$ & $25(9.2)$ & $12(5.7)$ & $15(8.5)$ \\
\hline $\begin{array}{l}\text { Paralysis/ataxia/numbness/ } \\
\text { tingling in extremities }\end{array}$ & $33(8.2)$ & $38(11.0)$ & 31 (9.9) & $34(12.6)$ & $23(11.0)$ & 29 (16.5) \\
\hline Pectoral angina/chest pain & $63(15.7)$ & 43 (12.4) & $35(11.1)$ & $29(10.7)$ & $24(11.5)$ & $17(9.7)$ \\
\hline Sweating (perspiration, diaphoresis) & $29(7.2)$ & $9(2.6)$ & $12(3.8)$ & $8(3.0)$ & $7(3.3)$ & $8(4.5)$ \\
\hline Syncope & $8(2.0)$ & $8(2.3)$ & $6(1.9)$ & $9(3.3)$ & 4 (1.9) & $2(1.1)$ \\
\hline Vertigo & 4 (1.0) & $0(0)$ & $2(0.6)$ & $1(0.4)$ & $1(0.5)$ & $1(0.6)$ \\
\hline
\end{tabular}

loss of appetite (56\%) were documented in $50 \%$ or more of these patients. Abdominal pain (35\%), depression/anxiety (43\%), dizziness/lightheadedness (30\%), edema (39\%), palpitations/tachycardia (34\%), and pectoral angina/chest pain (34\%) were documented in $30 \%$ or more of these patients. In patients who did not develop CIA, fatigue remained the most prevalent symptom ( $77 \%$ of the patients). Other than fatigue, only dyspnea/shortness of breath (41\%), nausea/ vomiting (43\%) and paralysis/ataxia/tingling in extremities $(33 \%)$ were noted in $30 \%$ or more of this study sample.

Table 4 shows the number and proportion of study patients with symptoms that occurred during each chemotherapy cycle. Again, fatigue is the predominant symptom documented throughout cycles for all patients. In patients who developed CIA, the proportion of patients experiencing the following symptoms was relatively stable across chemotherapy cycles: depression/anxiety, dizziness/lightheadedness, fatigue, pale skin, and sweating. The proportion of patients experiencing paralysis/ataxia/numbness/ tingling in extremities increased over cycles. For headache, loss of appetite, hypotension, and nausea/vomiting, the proportion of patients with symptom documentation was 


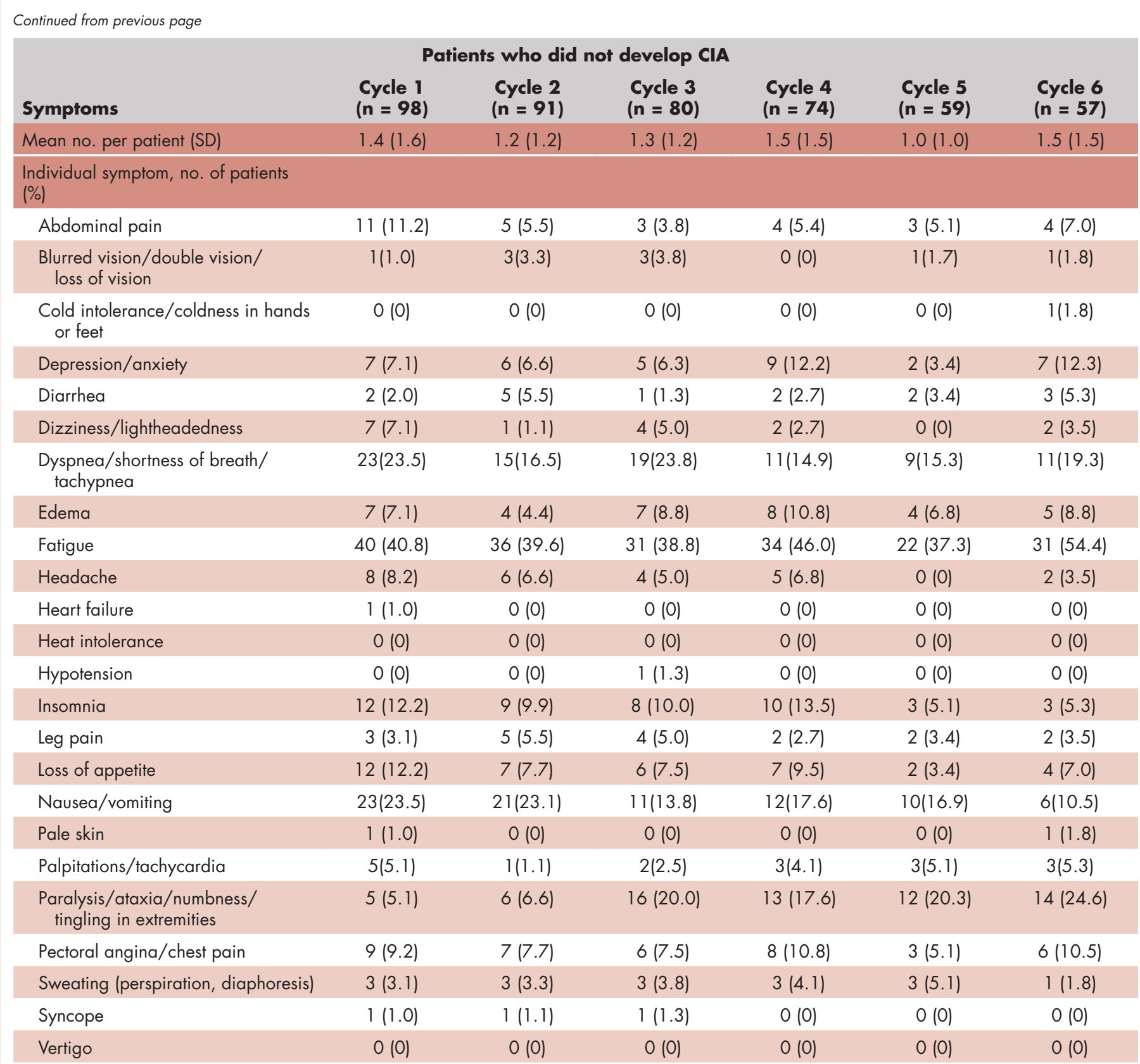

$\mathrm{CIA}$, chemotherapy-induced anemia

highest in cycle 1 , stabilizing in subsequent cycles (Table 4). In patients without CIA, the cycle-level prevalence of most of the symptoms did not increase over cycles, except for paralysis/ataxia/numbness or tingling in extremities. For insomnia, loss of appetite, and nausea/vomiting, the cycle-level prevalence dropped after the first cycle. There was no clear increasing trend of the mean number of symptoms per patient across chemotherapy cycles in both study samples (Table 4).

Table 5 shows the distribution of symptoms by anemia grade in patients who developed CIA. In general, the prevalence of symptoms increased with higher grades of anemia. The following symptoms especially have a clear increase in prevalence as the severity of anemia progressed: abdominal pain, depression, diarrhea, dizziness/lightheadedness, dyspnea, edema, fatigue, heart failure, headache, hypotension, insomnia, leg pain, loss of appetite, pale skin, palpitations, pectoral angina, and sweating. The mean number of symptoms per patient increased as CIA grade increased, from $3.6(\mathrm{SD}, 2.9)$ for grade $2 \mathrm{CIA}$ to $5.4(\mathrm{SD}, 3.5)$ for grades 
3 and 4 CIA (specifically, 5.3 [SD , 3.4] for grade 3 CIA and 6.4 [SD, 4.1] for grade 4 CIA; data not shown) (Table 5).

When stratified by gender, there are no material differences between men and women in most analyses. In men, the mean number of pre-existing symptoms was 1.7 (SD, 1.8) and 1.0 (SD, 1.2) for those with and without CIA, respectively $(P=.02)$. The mean number of symptoms that occurred during chemotherapy was 7.0 (SD, 3.4) and 4.2 (SD, 2.4), respectively $(P<.01)$. In women, the mean number of pre-existing symptoms was not statistically different in those with and without CIA (1.6 [SD, 2.2] and 1.3 [SD, 1.8], respectively; $P=.46)$. However, like in men, the mean number of symptoms that occurred during chemotherapy was significantly more in those with CIA (6.5 [SD, 3.3] and 4.0 [SD, 2.9], respectively; $P<.01)$. As in the overall analysis, there was no clear increasing trend of the number of symptoms per patients across chemotherapy cycles in both men and women, but the average number of symptoms increased as the CIA grade increased. For men, the mean number of symptoms per patient increased from 3.7 (SD, 3.0) for grade 2 CIA to $6.0(\mathrm{SD}, 3.5)$ for grades 3 and 4 CIA (data not shown). For women, the mean number of symptoms per patient increased from 3.6 (SD, 2.9) for grade 2 CIA to 4.7 (SD, 3.3) for grades 3 and 4 CIA (data not shown).

\section{Discussion}

In this study, we described the number and type of symptoms documented in the medical record notes among stage IV NHL, breast cancer, and lung cancer patients who did or did not develop CIA during chemotherapy. Patients who developed CIA had significantly greater numbers of different symptoms documented during chemotherapy than those who did not develop CIA (6.8 vs 4.1). This difinduced anemia
TABLE 5 Distribution of symptom by grade of anemia episodes for patients who developed chemotherapy-

\begin{tabular}{|c|c|c|c|}
\hline Symptoms & $\begin{array}{l}\text { Grade } 1^{\mathrm{a}} \\
(\mathrm{n}=297)\end{array}$ & $\begin{array}{l}\text { Grade } 2 \\
(n=355)\end{array}$ & $\begin{array}{l}\text { Grade } 3+4 \\
(n=119)\end{array}$ \\
\hline Mean no. of symptoms per patient (SD) & $1.8(1.9)$ & $3.6(2.9)$ & $5.4(3.5)$ \\
\hline \multicolumn{4}{|l|}{$\begin{array}{l}\text { By individual symptom, } \\
\text { no. of events with symptom (\%) }\end{array}$} \\
\hline Abdominal pain & $23(7.7)$ & $51(14.4)$ & $34(28.6)$ \\
\hline Blurred vision/double vision/loss of vision & $10(3.4)$ & $13(3.7)$ & $7(5.9)$ \\
\hline Cold intolerance/coldness in hands or feet & $3(1)$ & $5(1.4)$ & $5(4.2)$ \\
\hline Depression/anxiety & $20(6.7)$ & $80(22.5)$ & $40(33.6)$ \\
\hline Diarrhea & $14(4.7)$ & $54(15.2)$ & $30(25.2)$ \\
\hline Dizziness/lightheadedness & $23(7.7)$ & 47 (13.2) & $23(19.3)$ \\
\hline Dyspnea/ shortness of breath/tachypnea & $48(16.2)$ & $121(34.1)$ & $60(50.4)$ \\
\hline Edema & $25(8.4)$ & $72(20.3)$ & $46(38.7)$ \\
\hline Fatigue & $106(35.7)$ & $234(65.9)$ & $94(79)$ \\
\hline Headache & $20(6.7)$ & $33(9.3)$ & $19(16)$ \\
\hline Heart failure & $0(0)$ & $10(2.8)$ & $4(3.4)$ \\
\hline Heat intolerance & $1(0.3)$ & $3(0.8)$ & $0(0)$ \\
\hline Hypotension & $4(1.3)$ & $30(8.5)$ & $22(18.5)$ \\
\hline Insomnia & $21(7.1)$ & 47 (13.2) & $23(19.3)$ \\
\hline Leg pain & $21(7.1)$ & $34(9.6)$ & $12(10.1)$ \\
\hline Loss of appetite & $50(16.8)$ & $115(32.4)$ & $51(42.9)$ \\
\hline Nausea/vomiting & $55(18.5)$ & $115(32.4)$ & $39(32.8)$ \\
\hline Pale skin & $0(0)$ & $13(3.7)$ & $9(7.6)$ \\
\hline Palpitations/tachycardia & $16(5.4)$ & $63(17.7)$ & $41(34.5)$ \\
\hline $\begin{array}{l}\text { Paralysis/ataxia/numbness, tingling } \\
\text { in extremities }\end{array}$ & $30(10.1)$ & $40(11.3)$ & $15(12.6)$ \\
\hline Pectoral angina/chest pain & $27(9.1)$ & $57(16.1)$ & $42(35.3)$ \\
\hline Sweating (perspiration, diaphoresis) & $11(3.7)$ & $23(6.5)$ & $13(10.9)$ \\
\hline Syncope & $3(1)$ & $17(4.8)$ & $7(5.9)$ \\
\hline Vertigo & $0(0)$ & $6(1.7)$ & $3(2.5)$ \\
\hline \multicolumn{4}{|c|}{$\begin{array}{l}\text { although grade } 1 \text { anemia was not considered chemotherapy-induced anemia in this study, here, we presented symptoms } \\
\text { documented for grade } 1 \text { anemia in patients who developed chemotherapy-induced anemia (ie, grade } 2 \text { and higher) during } \\
\text { chemotherapy. }\end{array}$} \\
\hline
\end{tabular}

ference is clinically significant because most symptoms described in this study can be expected to have a negative impact on a patient's quality of life. In patients who developed CIA, fatigue was the most commonly documented symptom, noted for $90 \%$ of the study population. In addition to fatigue, many other symptoms were noted in a large proportion of patients. In contrast, in patients who did not develop CIA, only a few symptoms (including fatigue) were more commonly noted in this sample. We observed more symptoms in chemotherapy cycles with higher grades of anemia. Of the symptoms examined, abdominal pain, depression, diarrhea, dizziness/lightheadedness, dys- 
pnea, edema, fatigue, heart failure, headache, hypotension, insomnia, leg pain, loss of appetite, nausea/vomiting, pale skin, pectoral angina, sweating, and syncope particularly demonstrated a clearly increasing prevalence with declining $\mathrm{Hb}$ level. We also reported that patients who developed severe anemia (grades 3 and 4) experienced an average of 5 to 6 different symptoms at the time of the anemia episode. These data demonstrated a significant symptom burden in cancer patients with CIA seen in community-based oncology practices. Findings on the types of symptoms most commonly noted in various grades of CIA episodes provided some guidance for supportive care planning. As previous studies have shown a reduction in symptom burden after anemia treatment in patients with CIA, ${ }^{14-16}$ our results support the idea of early lab draws and active management of CIA in maintaining quality of life in cancer patients undergoing chemotherapy.

Our findings on the prevalence of fatigue are in line with other studies in the literature. Maxwell reported that the prevalence of fatigue was $80 \%$ to $96 \%$ in cancer patients. ${ }^{17}$ Cella and colleagues found that using FACT-General questionnaire, $75 \%$ of cancer patients reported fatigue. ${ }^{11}$ The comparability of our estimate and those found in studies based on patient self-report offered some assurance of the validity of assessing symptom prevalence through physician record notes. In addition to fatigue, we described prevalence of 23 additional symptoms, most of which have not been extensively studied in the literature. Gabrilove and colleagues found that a substantial proportion of patients with CIA had moderate to severe score for lack of appetite (36\%) and disturbed sleep (41\%) using the MDASI. ${ }^{10}$ The prevalence of loss of appetite and insomnia was around $50 \%$ and 25\%, respectively, in our study samples. A 2013 systematic review of 21 multinational studies reported the pooled prevalence of several nonfatigue symptoms in cancer patients including headache (23\%), sleep disturbance/ insomnia (49\%), appetite changes (45\%), nausea/vomiting (26\%), diarrhea (15\%), depression (34\%), dyspnea (44\%), dizziness (26\%), numbness/tingling (42\%), edema (14\%), and sweating (28\%). ${ }^{18}$ Our prevalence estimates in patients with CIA for most of these symptoms were higher, likely because Reilly and colleagues used source studies that included any cancer patients undergoing treatment and not just those with CIA. Our findings on the increased symptom burden in patients who experienced episodes of advanced anemia compared with patients with mild anemia were also consistent with the literature. To this end, several studies using MDASI or the FACT-An reported differential symptom burdens by $\mathrm{Hb}$ level based on patient self-report, ${ }^{10,11,19}$ including data on improvement in symptom burden and quality of life after anemia was amended with the use of ESA. ${ }^{20,21}$

We found that the number of pre-existing symptoms was significantly higher in patients who went on to develop
CIA than in patients who did not develop CIA. Specifically, fatigue, loss of appetite, and pale skin before chemotherapy seemed to be significantly more common in patients who went on to develop CIA. This finding suggested that presentation of these symptoms before chemotherapy initiation may be a predictor for developing moderate or severe anemia during treatment. This is a novel hypothesis, as no studies have evaluated the relationship between pretreatment symptom and risk of CIA. However, our study was not designed to address this specific question. Additional investigation is needed to further shed light on whether the occurrence of anemia-related symptoms in nonanemic patients can be used to effectively risk-stratify patients for subsequent CIA.

Contrary to our expectation, the prevalence of most symptoms did not clearly increase as chemotherapy progressed. There are several possible explanations to this phenomenon, with the most likely being related to reporting of anemia-related symptoms. For example, patients might stop reporting the same symptom repeatedly or become adjusted to the new Hb levels, leading to less symptom manifestation. Clinicians may also be less likely to ask about symptoms in later treatment cycles and/or to document chronic symptoms. Several symptoms were rarely documented altogether, such as cold intolerance, heat intolerance, heart failure, and vertigo. Symptoms reported in earlier cycles could also be managed successfully. Another possible explanation is differential loss of follow-up. Patients who experienced severe adverse events or symptoms may terminate treatment prematurely. Thus, symptom burden found toward later cycles may not represent the true symptom burden should everyone who initiated the chemotherapy treatment complete all planned cycles.

\section{Limitations}

In addition to the limitations already discussed, there are several others that should be considered when interpreting our results. We did not have a consistent measure of symptom severity in the medical records. Duration of symptoms was also often poorly documented by physicians. Therefore, our results are not directly comparable with studies such as the MDASI that incorporate severity or duration in their prevalence measure. There may also be "reporting bias" by the clinicians owing to different perceived levels of severity or clinical relevance of the different symptoms. As a result, some symptoms may be underdocumented, leading to undercounting.

We also did not distinguish the exact cause of the symptoms (ie, owing to anemia, cancer, chemotherapy itself, or other chemotherapy-induced complications), as it was not possible to reliably ascertain the cause from record review. Furthermore, symptom assessment was not separately performed for grade 4 anemia because of the small number of events in the study population. We also did not plan to 
evaluate the impact of anemia treatment on symptom burden, as our goal was to comprehensively describe a wide spectrum of symptoms experienced by patients with different $\mathrm{Hb}$ levels. However, previous studies have shown the benefit of treatments that correct CIA in symptom management. ${ }^{14-16}$ Finally, this study does not inform about the relative importance of these symptoms to patients' quality of life. To this end, a qualitative study found fatigue, shortness of breath, and lightheadedness/dizziness to be the most important symptoms ranked by patients with CIA. ${ }^{22}$

Despite the potential limitations, our study has several important strengths. In addition to fatigue, patients with CIA suffer from a wide range of other anemia-related symptoms, but data on the prevalence of these symptoms have been lacking. To our knowledge, this is among the first studies that collected data on a comprehensive list of

\section{References}

1. Barrett-Lee PJ, Ludwig H, Birgegård G, et al. Independent risk factors for anemia in cancer patients receiving chemotherapy: results from the European Cancer Anaemia Survey. Oncology. 2006;70(1):34-48.

2. Kitano T, Tada H, Nishimura T, et al. Prevalence and incidence of anemia in Japanese cancer patients receiving outpatient chemotherapy. Int J Hematol. 2007;86(1):37-41.

3. Birgegård G, Aapro MS, Bokemeyer C, et al. Cancer-related anemia: pathogenesis, prevalence and treatment. Oncology. 2005;68(Suppl 1):3-11.

4. Harper P, Littlewood T. Anaemia of cancer: impact on patient fatigue and long-term outcome. Oncology. 2005;69(Suppl 2):2-7.

5. Nieboer P, Buijs C, Rodenhuis S, et al. Fatigue and relating factors in high-risk breast cancer patients treated with adjuvant standard or high-dose chemotherapy: a longitudinal study. J Clin Oncol. 2005;23(33):8296-8304.

6. Bremberg ER, Brandberg Y, Hising C, Friesland S, Eksborg S. Anemia and quality of life including anemia-related symptoms in patients with solid tumors in clinical practice. Med Oncol. 2007;24(1):95-102.

7. Hofman M, Ryan JL, Figueroa-Moseley CD, Jean-Pierre P, Morrow GR. Cancer-related fatigue: the scale of the problem. Oncologist. 2007;12(Suppl 1):4-10.

8. Cleeland CS. Symptom burden: multiple symptoms and their impact as patient-reported outcomes. J Natl Cancer Inst Monogr. 2007(37):16-21.

9. Yellen SB, Cella DF, Webster K, Blendowski C, Kaplan E. Measuring fatigue and other anemia-related symptoms with the Functional Assessment of Cancer Therapy (FACT) measurement system. J Pain Symptom Manage. 1997;13(2):63-74.

10. Gabrilove JL, Perez EA, Tomita DK, Rossi G, Cleeland CS. Assessing symptom burden using the M.D. Anderson symptom inventory in patients with chemotherapy-induced anemia: results of a multicenter, open-label study (SURPASS) of patients treated with darbepoetin-alpha at a dose of 200 microg every 2 weeks. Cancer. 2007;110(7):1629-1640.

11. Cella D. The Functional Assessment of Cancer Therapy-Anemia (FACT-An) scale: a new tool for the assessment of outcomes in can- symptoms and provided detailed analysis by chemotherapy cycle and anemia grade. The combined use of KPSC's clinical databases and medical record review allowed us to provide detailed characterization of the study population in terms of their treatment history, history of comorbidities, and laboratory data.

\section{Conclusions}

Our data provide physicians a comprehensive picture of prevalence of various types of symptoms and how symptom burden evolves as chemotherapy cycle and anemia severity progress. High-grade CIA correlates with an increased symptom burden. Such an understanding can be crucial in facilitating supportive care planning by helping physicians anticipate the timing and proactively determine the management approach of chemotherapy-related anemia and its symptoms.

cer anemia and fatigue. Semin Hematol. 1997;34(3 Suppl 2):13-19.

12. Koebnick C, Langer-Gould AM, Gould MK, et al.

Sociodemographic characteristics of members of a large, integrated health care system: comparison with US Census Bureau data. Perm J. 2012;16(3):37-41.

13. Groopman JE, Itri LM. Chemotherapy-induced anemia in adults: incidence and treatment. J Natl Cancer Inst. 1999;91(19):1616-1634.

14. Gilreath JA, Stenehjem DD, Rodgers GM. Diagnosis and treatment of cancer-related anemia. Am J Hematol. 2014;89(2):203-212.

15. Rizzo JD, Somerfield MR, Hagerty KL, et al. Use of epoetin and darbepoetin in patients with cancer: 2007 American Society of Clinical Oncology/American Society of Hematology clinical practice guideline update. J Clin Oncol. 2008;26(1):132-149.

16. Bohlius J, Tonia T, Nüesch E, et al. Effects of erythropoiesis-stimulating agents on fatigue- and anaemia-related symptoms in cancer patients: systematic review and meta-analyses of published and unpublished data. Br J Cancer. 2014;111(1):33-45.

17. Maxwell MB. When the cancer patient becomes anemic. Cancer Nurs. 1984;7(4):321-326.

18. Reilly CM, Bruner DW, Mitchell SA, et al. A literature synthesis of symptom prevalence and severity in persons receiving active cancer treatment. Support Care Cancer. 2013;21(6):1525-1550.

19. Crawford J, Cella D, Cleeland CS, et al. Relationship between changes in hemoglobin level and quality of life during chemotherapy in anemic cancer patients receiving epoetin alfa therapy. Cancer. 2002;95(4):888-895.

20. Mouysset JL, Freier B, van den Bosch J, et al. Hemoglobin levels and quality of life in patients with symptomatic chemotherapy-induced anemia: the eAQUA study. Cancer Manag Res. 2016;8:1-10.

21. Vansteenkiste J, Pirker R, Massuti B, et al. Double-blind, placebo-controlled, randomized phase III trial of darbepoetin alfa in lung cancer patients receiving chemotherapy. J Natl Cancer Inst. 2002;94(16):1211-1220.

22. Kleinman L, Benjamin K, Viswanathan $\mathrm{H}$, et al. The anemia impact measure (AIM): development and content validation of a patientreported outcome measure of anemia symptoms and symptom impacts in cancer patients receiving chemotherapy. Qual Life Res. 2012;21(7):1255-1266. 